\title{
Análise de Capacidade e Desempenho da Tecnologia de Transmissão Rádio IP-OFDMA
}

\author{
Adriano A. Goes, J. Sindi Yamamoto e Omar C. Branquinho
}

\begin{abstract}
Resumo--Neste trabalho é apresentada uma análise de capacidade e de desempenho da tecnologia de transmissão rádio IP-OFDMA, submetida recentemente pelo IEEE e WiMAX Forum à ITU-T como uma nova interface rádio IMT-2000, também conhecida como 3G. A análise é realizada tanto analiticamente como por simulação, utilizando-se de parâmetros sistêmicos do IP-OFDMA e modelo de propagação "shadwing". Os resultados desta análise mostram que o IP-OFDMA, embora atenda sob determinadas condições os requisitos do IMT-2000, pode ter a sua capacidade e desempenho comprometidos por usuários situados na periferia da célula.
\end{abstract}

\section{Palavras chaves-IP-OFDMA, WiMAX, IMT-2000}

Abstract-This paper presents an analysis of capacity and performance of the IP-OFDMA radio transmission technology, submitted recently by the IEEE and WiMAX Forum to the ITU-T as a new IMT-2000 interface radio, also known as 3G. The analysis is carried through analytically as well as by simulation, using the systemic parameters of the IP-OFDMA and the shadowing propagation model. The results of the analysis show that IP-OFDMA even so attends the IMT-2000 requirements under some conditions, it can have the capacity and performance compromised by the users in the periphery of the cell.

Key words-- IP-OFDMA, WiMAX, IMT-2000

\section{INTRODUÇÃO}

A tecnologia WiMAX fixo, baseado no padrão IEEE 802.16d (ou IEEE 802.16-2004) ratificado em julho de 2004 [1], tem-se consolidado como a principal tecnologia para WMAN(“Wireless Metropolitan Área Network”). Em janeiro de 2006, foram certificados os primeiros equipamentos WiMAX fixo, também denominado WiMAX “nomádico", pelo Cetecom na Espanha. Anteriormente, em dezembro de 2005, o IEEE já havia ratificado também o padrão IEEE 802.16e (ou IEEE 802.16-2005) [2]. Este novo padrão consiste

\footnotetext{
Adriano A. Góes e Omar Carvalho Branquinho, Departamento de Engenharia Elétrica, Pontifícia Universidade Católica de Campinas, Campinas-SP, Brasil J. Sindi Yamamoto, Innovatech Telecom, Galleria Office Park: Av. Selma Parada, 201-Bloco 3 - Sala 301, Jardim Madalena - Campinas - SP, Brasil http://www.innovatechtelecom.com e-mail: innovatech@innovatechtelecom.com
}

de um complemento ao padrão IEEE 802.16d, adicionando características e funcionalidades necessárias para suportar a mobilidade. A tecnologia utilizando o padrão IEEE 802.16e é conhecida como WiMAX móvel. A interface aérea do WiMAX móvel utiliza a técnica de acesso OFDMA (Orthogonal Frequency Division Multiple Access) que melhora o desempenho em ambientes com múltiplos percursos e sem linha de visada. Adicionalmente, no padrão IEEE 802.16e é introduzida a técnica de acesso S-OFDMA ("Scalable OFDMA") para suportar canais com largura de banda escalonada de 1,25 a $20 \mathrm{MHz}$ [3]. O perfil sistêmico do WiMAX móvel permite a configuração de sistemas móveis baseado em um conjunto de características básicas comuns que asseguram funcionalidades para as MS ("Mobile Station”) e BS ("Base Sation”) totalmente inter-operáveis. Alguns elementos do perfil da BS são especificados como opcionais para prover uma flexibilidade adicional de utilização, dependendo de cenários de aplicação que podem necessitar de diferentes configurações otimizadas em termos de capacidade ou cobertura. Assim, a rede WiMAX móvel pretende ser uma solução de banda larga sem fio que possibilita a convergência das redes de banda larga fixa e móvel através de uma tecnologia de acesso rádio de banda larga, grande área de cobertura e uma arquitetura de rede flexível.

Em novembro de 2006, o IEEE em combinação com WiMAX Forum, propôs que uma nova tecnologia de transmissão rádio terrestre baseada no padrão IEEE 802.16e, chamada IP-OFDMA, fosse incluída na recomendação ITU-R M.1457 ("Detailed specifications of the radio interfaces of International Mobile Telecommunications-2000 (IMT-2000)”), de acordo com os procedimentos da ITU-R para a adição de novas tecnologias de transmissão rádio[4]. Baseado em sua própria avaliação realizada segundo a metodologia descrita na Recomendação ITU-R M.1225 ("Guidelines for Evaluation of Radio Transmission Technologies for IMT-2000”), o WiMAX Forum acredita que o IP-OFDMA atende os requisitos e objetivos do IMT-2000, também conhecido como 3G. Sob este contexto, no presente trabalho é feita uma análise de capacidade e de desempenho da tecnologia de transmissão rádio IP-OFDMA. A análise é realizada tanto analiticamente como por simulação, utilizando-se de parâmetros sistêmicos do IP-OFDMA [5] e modelo de propagação "shadowing” [6]. Para tanto, o trabalho encontra-se organizado da seguinte forma: na seção II são apresentadas as principais características e parâmetros sistêmicos do IP-OFDMA. Nas seções III e IV são apresentadas, respectivamente, as análises de capacidade e 
de desempenho realizadas. Finalmente, as conclusões são apresentadas na seção V.

\section{TeCnOlogia de Transmissão RÁdio IP-OFDMA}

\section{II.1. INTRODUÇÃo}

O OFDM é uma técnica de multiplexação que divide a largura de banda disponível em múltiplas freqüências ortogonais, denominadas sub-portadoras, como ilustrado na figura 1. Assim, no sistema OFDM, uma sequiência de bits de dados de entrada é convertida, através de um conversor série/paralelo, em diversas sub-seqüências de bits, obtendo-se uma redução na taxa de dados em cada sub-seqüência e, portanto, um aumento na duração do símbolo. Cada subseqüência de bits é modulada e transmitida sobre uma subportadora ortogonal. $\mathrm{O}$ aumento na duração do símbolo melhora a robustez ao espalhamento temporal ("delay spread") do sinal OFDM. Adicionalmente, a introdução de um prefixo cíclico, denominado CP ("Cyclic Prefix"), pode eliminar completamente a interferência inter-simbólica na medida em que a duração do $\mathrm{CP}$ for maior do que o espalhamento temporal do canal [7]. A técnica OFDM explora a diversidade de freqüência do canal com múltiplos percursos codificando e embaralhando a informação através das sub-portadoras antes da transmissão. O sinal OFDM é obtida convertendo-se o conjunto das sub-portadoras para o domínio do tempo através da transformada inversa rápida de Fourier ("IFFT:Inverse Fast Fourier Transform”). Assim, no sistema OFDM, os recursos de rádio são disponíveis no domínio do tempo por meio de símbolos OFDM, e no domínio da freqüência por meio das sub-portadoras. Os recursos no domínio do tempo e freqüência podem ser organizados em sub-canais para alocação aos usuários. A técnica OFDMA (“Orthogonal Frequency Division Multiple Access”) é um esquema de acesso múltiplo/multiplexação que realiza a operação OFDM de seqüências de dados de múltiplos usuários nos sub-canais do enlace direto, DL (“down link”) e acesso múltiplo no enlace reverso, UL ("up link”) através dos sub-canais do enlace reverso.

A estrutura de símbolo do OFDMA consiste de 3 tipos de sub-portadoras:

- Sub-portadora de dados: para a transmissão de dados.

- Sub-portadora piloto: para estimação e sincronização

- Sub-portadoras “Null”: não transmitem nenhuma informação, sendo usados para banda de guarda e sub-portadora zero $\mathrm{Hz}$.

\section{II.2. ESTRUTURA DE QUADRO}

A figura 2 mostra um exemplo de estrutura de quadro TDD ("Time Division Multiplex") do IP-OFDMA, onde a dimensão horizontal corresponde ao tempo e a dimensão vertical corresponde à freqüência [8]. Assim, o eixo vertical da estrutura de quadro é dividido em $L$ sub-portadoras ortogonais.

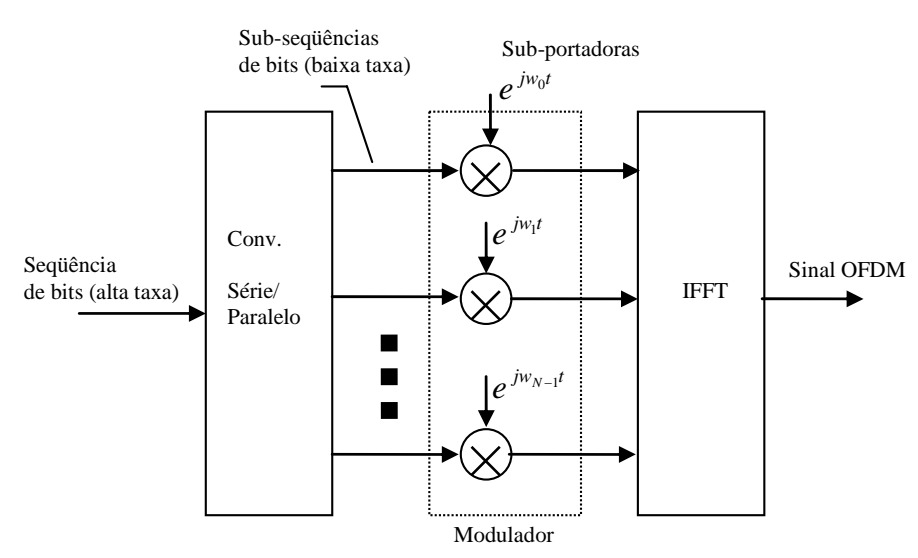

Fig.1. Arquitetura básica de um sistema OFDM

O eixo horizontal é dividido em intervalos de tempo correspondente a um símbolo, sendo $M$ símbolos no DL e $N$ símbolos no UL. Os $M$ símbolos no sentido direto e os $N$ símbolos no sentido reverso formam, respectivamente, os subquadros no sentido direto (sub-quadro DL) e no sentido reverso (sub-quadro UL). Depois do sub-quadro DL, existe uma banda de guarda denominado TTG ("Transmit/Receive Transition Gap"). Da mesma forma, depois do sub-quadro UL, existe uma banda de guarda denominado RTG ("Receive/Transmit Transition Gap").

O primeiro símbolo no sub-quadro DL é o preâmbulo, que é seguido pelos cabeçalhos de controle e sinalização denominados FCH (Frame Control Header), DL-MAP ("Media Access Protocol”) e UL-MAP.

O preâmbulo é usado para sincronização. O FCH provê informação de configuração do quadro, tais como o comprimento da mensagem MAP, esquema de codificação e sub-canais disponíveis. O DL-MAP e UL-MAP são cabeçalhos importantes que mostram como os surtos de dados dos usuários estão alocados na área de dados dos sub-quadros DL e UL, respectivamente. Cada terminal móvel conhece a localização dos surtos de dados destinados ao mesmo através da leitura do DL-MAP. Da mesma forma, o terminal móvel sabe onde carregar os seus dados no UL através do UL-MAP. Os surtos de dados do usuário no DL são alocados depois do MAP. Um sub-quadro UL inicia com os canais de controle CQICH ("Channel Quality Indicator Channel”), ACKCH ("Acknowledge Channel") e "Ranging Channels". O canal CQICH é usado para transmitir a informação de estado do canal da MS para a BS. O canal ACKCH é usado pelas MSs para transmitir a informação de confirmação de recebimento (ACK) da mensagem DL HARQ (Hybrid Automatic Repeat reQuest). O "ranging channel" é usado para requisição de largura de banda e ajuste de potência, frequiência e temporização. Depois das informações de controle, surtos de dados UL são alocados de acordo com o UL-MAP. Cada surto de dados consiste de um ou mais "slots" que é a unidade mínima de alocação possível. As estruturas dos "slots" são 
diferentes para cada método de sub-canalização: DL PUSC ("Partial usage of sub-channel"): 2 símbolos OFDMA; DL FUSC ("Full usage of sub-channles”): 1 símbolo OFDMA; UL PUSC ("Partial usage of sub-channels") e DL/UL AMC(“Adaptive modulation and coding”): 2, 3 ou 6 símbolos OFDMA.

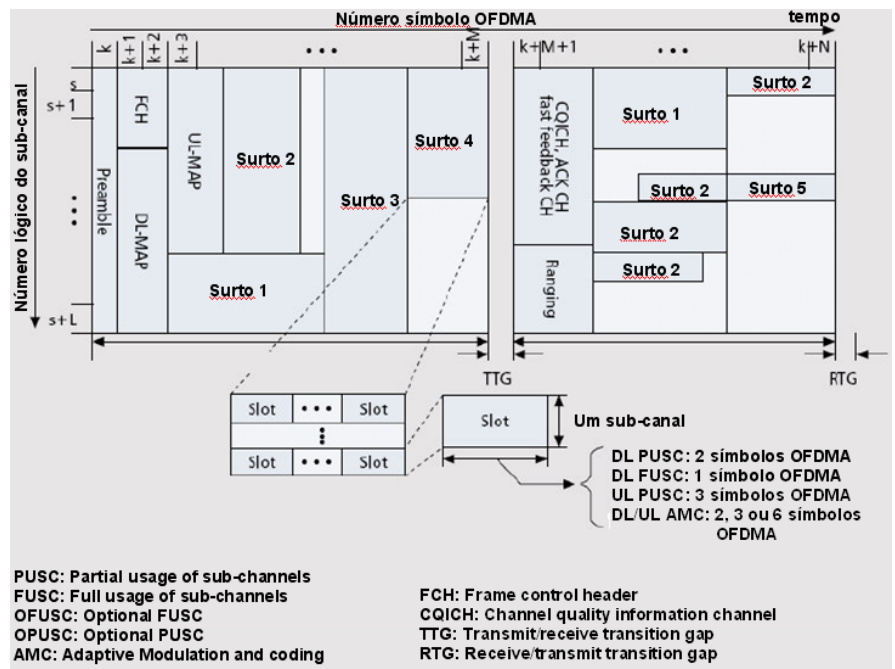

Fig. 2. Exemplo de estrutura de quadro IP-OFDMA [8]

O modo TDD permite ajustar a razão "downlink/uplink" (DL/UL) para suportar tráfegos simétricos (razão DL/UL=1:1) e assimétricos (DL:UL > 1:1). Como mostrado na tabela I, 0 número de símbolos OFDM DL:UL recomendado permite implementar de modo flexível uma faixa de razão de tráfego DL/UL de 3:1 a 1:1 [5].

TABELA I

NúMERO DE SÍMBOLOS OFDM NO DL E UL

\begin{tabular}{|l|l|}
\hline \multicolumn{1}{|c|}{ Descrição } & \multicolumn{1}{c|}{ Valores DL:UL } \\
\hline Número de símbolos & $(35: 12),(34: 13),(33:$ \\
OFDM no DL e UL para & $14),(32: 15),(31: 16)$, \\
larguras de banda de 5 and & $(30: 17),(29: 18),(28:$ \\
$10 \mathrm{MHz}$ & $19),(27: 20),(26: 21)$ \\
\hline
\end{tabular}

\section{II.3. PARÂMETROS E VALORES DO IP-OFDMA}

O IP-OFDMA pode operar com uma largura de banda de 5 $\mathrm{MHz}$ ou $10 \mathrm{MHz}$. A escalabilidade é suportada ajustando-se o tamanho da FFT e mantendo-se o espaçamento entre as subportadoras em 10,94 kHz e a duração do símbolo em 102,9 $\mu$ s. Os parâmetros e valores do IP-OFDMA são mostrados na tabela II [5].

\section{II.4 ESQUEMAS DE MODULAÇÃo E CODIFICAÇÃo}

No IP-OFDMA, codificação e modulação adaptativas, HARQ, CQICH e tecnologias de múltiplas antenas provêem melhoria de cobertura e capacidade. Assim, no IP-OFDMA as modulações QPSK, 16 QAM e 64 QAM são mandatórias no DL. No UL, 64 QAM é opcional. Também são suportados os códigos convolucional (CC) e Turbo (CTC) com taxa de codificação variável e repetição. Na tabela III são mostrados os esquemas de modulação e codificação suportados no IPOFDMA [5].

TABELA II

PARÂMETROS E VALORES DO IP-OFDMA

\begin{tabular}{|c|c|c|c|c|}
\hline Parametros & Downlink & Uplink & Downlink & Uplink \\
\hline Largura de banda & \multicolumn{2}{|c|}{$5 \mathrm{MHz}$} & \multicolumn{2}{|c|}{$10 \mathrm{MHz}$} \\
\hline Freq. de amostragem, $F_{p}$ & \multicolumn{2}{|c|}{$5.6 \mathrm{MHz}$} & \multicolumn{2}{|c|}{$11.2 \mathrm{MHz}$} \\
\hline Tamanho da FFT & \multicolumn{2}{|c|}{512} & \multicolumn{2}{|c|}{1024} \\
\hline Sub-portadoras "Null” & 92 & 104 & 184 & 184 \\
\hline Sub-portadoras Piloto & 60 & 136 & 120 & 280 \\
\hline Sub-portadoras de dados & 360 & 272 & 720 & 560 \\
\hline Número de sub-canais & 15 & 17 & 30 & 35 \\
\hline $\begin{array}{l}\text { Espaçamento entre sub- } \\
\text { portadoras }\end{array}$ & \multicolumn{4}{|c|}{$10.94 \mathrm{kHz}$} \\
\hline $\begin{array}{l}\text { Duração útil do símbolo } \\
\left(T_{b}=1 / f\right)\end{array}$ & \multicolumn{4}{|c|}{$91,4 \mu \mathrm{s}$} \\
\hline $\begin{array}{l}\text { Tempo de guarda }\left(\mathrm{T}_{\mathrm{g}}\right. \\
\left.=\mathrm{T}_{\mathrm{b}} / 8\right)\end{array}$ & \multicolumn{4}{|c|}{$11,4 \mu \mathrm{s}$} \\
\hline $\begin{array}{l}\text { Duração do símbolo }\left(\mathrm{T}_{\mathrm{s}}\right. \\
\left.=\mathrm{T}_{\mathrm{b}}+\mathrm{T}_{\mathrm{g}}\right)\end{array}$ & \multicolumn{4}{|c|}{$102,9 \mu \mathrm{s}$} \\
\hline Duração do quadro & \multicolumn{4}{|c|}{$5 \mathrm{~ms}$} \\
\hline Símbolos OFDM/quadro & \multicolumn{4}{|c|}{48 (including 1.6 symbols for TTG/RTG) } \\
\hline $\begin{array}{l}\text { Símbolos OFDM de } \\
\text { dados }\end{array}$ & \multicolumn{4}{|c|}{ 48-Overhead } \\
\hline
\end{tabular}

TABELA III

ESQUEMAS DE CODIFICAÇÃO E MODULAÇÃO SUPORTADOS PELO IP-OFDMA

\begin{tabular}{|l|l|l|l|}
\hline \multicolumn{2}{|c|}{} & \multicolumn{1}{|c|}{ DL } & \multicolumn{1}{c|}{ UL } \\
\hline \multicolumn{2}{|c|}{ Modulação } & $\begin{array}{l}\text { QPSK, 16QAM, } \\
\text { 64QAM }\end{array}$ & $\begin{array}{l}\text { QPSK,16QAM, } \\
\text { 64QAM opcional) }\end{array}$ \\
\hline \multirow{2}{*}{ Razão } & CC & $1 / 2,2 / 3,3 / 4$ & $1 / 2,2 / 3,3 / 4$ \\
\cline { 2 - 5 } & CTC & $1 / 2,2 / 3,3 / 4,5 / 6$ & $1 / 2,2 / 3,5 / 6$ \\
\cline { 2 - 5 } & Repetição & $x 2, x 4, x 6$ & $x 2, x 4, x 6$ \\
\hline
\end{tabular}

\section{II.5. Provimento de QoS}

Os mecanismos de alocação de recursos e a granularidade de recursos do IP-OFDMA permite atender os requisitos de QoS para uma grande gama de serviços de dados e aplicações. Em [5] são definidos 5 categorias de QoS: UGS (Unsolicited Grant Service), rtPS(Real-Time Packet Service), ErtPS (Extended Real-Time Packet Service), nrtPS(Non-Real-Time Packet Service) e BE (Best-Effort Service). Na tabela IV têm-se o resumo de serviços de dados e aplicações bem como os requisitos de QoS para cada uma das categorias. 
TABELA IV

APLICAÇÕES IP-OFDMA E QUALIDADE DE SERVIÇO

\begin{tabular}{|l|l|l|}
\hline Categoria de QoS & Aplicações & Especificação de QoS \\
\hline $\begin{array}{l}\text { UGS: Unsolicited Grant } \\
\text { Service }\end{array}$ & VoIP & $\begin{array}{l}\text { Taxa sustentada máxima } \\
\text { Tolerância de latência } \\
\text { maxima. } \\
\text { Tolerância de “jitter". }\end{array}$ \\
\hline $\begin{array}{l}\text { rtPS: Real-Time Packet } \\
\text { Service }\end{array}$ & $\begin{array}{l}\text { "Streaming” de } \\
\text { Audio ou Vídeo }\end{array}$ & $\begin{array}{l}\text { Minimum Reserved Rate } \\
\text { Maximum Sustained Rate } \\
\text { Maximum Latency Tolerance } \\
\text { Traffic Priority }\end{array}$ \\
\hline $\begin{array}{l}\text { ErtPS: Extended Real- } \\
\text { Time Packet Service }\end{array}$ & $\begin{array}{l}\text { Voz com } \\
\text { detecção de } \\
\text { atividade } \\
\text { (VoIP) }\end{array}$ & $\begin{array}{l}\text { Taxa reservada mínima } \\
\text { Taxa sustentada máxima } \\
\text { Tolerância de latência } \\
\text { máxima } \\
\text { Tolerância de “jitter" } \\
\text { Prioridade de tráfego }\end{array}$ \\
\hline $\begin{array}{l}\text { nrtPS: Non-Real-Time } \\
\text { Packet Service }\end{array}$ & $\begin{array}{l}\text { File Transfer } \\
\text { Protocol (FTP) }\end{array}$ & $\begin{array}{l}\text { Taxa reservada mínima } \\
\text { Taxa sustentada máxima } \\
\text { Prioridade de tráfego }\end{array}$ \\
\hline BE: Best-Effort Service & $\begin{array}{l}\text { Transferência } \\
\text { de dado, Web } \\
\text { Browsing, etc. }\end{array}$ & $\begin{array}{l}\text { Taxa sustentada maxima } \\
\text { Prioridade de tráfego }\end{array}$ \\
\hline
\end{tabular}

\section{ANÁliSE DE CAPACIDADE}

\section{III.1. TAXA DE BITS POR CANAL DE RF}

A taxa de bits por RF depende das seguintes configurações do IP-OFDMA: a) Largura de banda: $10 \mathrm{MHz}$ ou $5 \mathrm{MHz}$; b) Razão DL:UL; c) Esquema de modulação e codificação; d) Reuso de freqüências. O uso dos esquemas de modulação e codificação, mostrados nas tabela III, depende do nível do sinal na entrada do receptor OFDMA. Em termos de reuso de freqüências, são considerados duas configurações: a) (1:3:3) 1 BS por "cluster", 3 setores por BS e 3 canais de RF; b) (1:3:1) - 1 BS por "cluster", 3 setores por BS e 1 canal de RF. A figura 3 ilustra as duas configurações de reuso considerados.
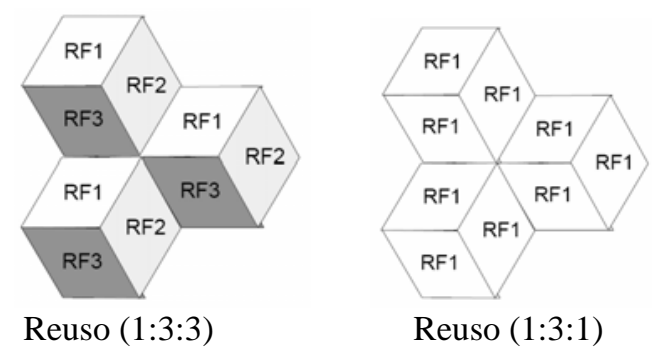

Fig. 3. Reuso de freqüências.

A taxa de bits por RF no enlace direto pode ser calculado pela seguinte expressão:

$$
t b_{-} d l=\frac{s p_{-} d l}{d} \times n b \frac{s_{-} d l}{s},
$$

onde: $s p \_d l$ : quantidade de sub-portadoras no DL

$d$ : duração do símbolo

$n b$ : quantidade de bits/sub-portadora/símbolo

$s \_d l$ : quantidade de símbolos de dado no DL

$s:$ quantidade de símbolos por quadro

Analogamente, a taxa de bits por RF no enlace reverso é dado pela expressão:

$$
t b_{-} u l=\frac{s p_{-} u l}{d} \times n b \frac{s_{-} u l}{s},
$$

onde sp_ul e s_ul são a quantidade de sub-portadoras e símbolos de dados no enlace reverso, respectivamente.

A quantidade de símbolos de dados no DL, s_dl, é igual a:

$$
s_{-} d l=t s_{-} d l \text {-overhead },
$$

onde $t s \_d l$ é a quantidade de símbolos alocados no DL e o overhead corresponde a quantidade de símbolos utilizados pelo preâmbulo, FCH, DL_MAP e UL_MAP. O preâmbulo e o FCH utilizam uma quantidade fixa de 1 símbolo e 0,267 símbolos, respectivamente. O overhead devido ao DL_MAP e UL_MAP consiste de uma parte fixa e de uma parte variável com o número de MSs ativos em um quadro. Tipicamente, a parte fixa do DL_MAP corresponde a 104 bits e a parte variável a $N \times 60$ bits, onde $N$ é o número de usuários ou MSs ativos em um quadro. No caso do UL_MAP, a parte fixa corresponde tipicamente a 60 bits e a parte variável a $N \times 32$ bits [9]. Desta forma, obtém-se:

$$
s_{-} d l=t s_{-} d l-[1,267+(164+N \times 92) /(720 * n b)]
$$

O overhead no caso do UL é de 3 símbolos. Portanto, tem-se que:

$$
s_{-} u l=t s_{-} d l-3
$$

Desta forma, a taxa de bits por RF no DL depende do número de usuários ativos, $N$, em um quadro, pois a quantidade de símbolos de dados diminui com a alocação dos mesmos para DL_MAP à medida que cresce o número de usuários. Assim, por exemplo, para a modulação QPSK, 1/2 CTC, 6x, DL:UL=(35:12) e reuso de freqüência (1:3:1), a quantidade de símbolos disponíveis para dados diminui 0,751 símbolos por usuário ativo. Assim, a quantidade de símbolos de dados tornase igual a:

$$
s_{-} d l=32,40-N \times 0,751
$$

Na tabela $\mathrm{V}$ são mostradas as taxa de bits por RF considerando-se apenas um usuário $(N=1)$ ativo em um quadro para vários esquemas de codificação e modulação, configuração DL:UL e reuso de freqüências. A taxa de bits é maior para o reuso de freqüência (1:3:1) onde, entretanto, o 
nível de interferência é significativo. No caso de reuso de freqüências (1:3:3), o nível de interferência é menor e a capacidade é 3 vezes menor em relação ao reuso de freqüência (1:3:1).

\section{TABELA V}

TAXA DE BITS POR RF PARA $\mathrm{N}=1$

\begin{tabular}{|c|c|c|c|c|c|c|}
\hline \multirow[t]{2}{*}{ DL:UL } & \multirow[t]{2}{*}{ Modulação } & \multirow[t]{2}{*}{$\begin{array}{l}\text { Reuso de } \\
\text { freqüências }\end{array}$} & \multicolumn{2}{|c|}{$\begin{array}{c}\text { Taxa de bits DL } \\
\text { (Mbps) }\end{array}$} & \multicolumn{2}{|c|}{$\begin{array}{l}\text { Taxa de bits } \\
\text { UL (Mbps) }\end{array}$} \\
\hline & & & $\begin{array}{c}10 \\
\mathrm{MHz}\end{array}$ & $\begin{array}{c}5 \\
\mathrm{MHz}\end{array}$ & $\begin{array}{c}10 \\
\mathrm{MHz}\end{array}$ & $\begin{array}{c}5 \\
\mathrm{MHz}\end{array}$ \\
\hline \multirow[t]{8}{*}{$44: 3$} & \multirow{2}{*}{$\begin{array}{l}\text { QPSK, } 1 / 2 \\
\text { CTC, } 6 \mathrm{x}\end{array}$} & $(1: 3: 3)$ & 0,353 & 0,177 & 0 & 0 \\
\hline & & $(1: 3: 1)$ & 1,060 & 0,530 & 0 & 0 \\
\hline & \multirow{2}{*}{$\begin{array}{l}\text { 16 QAM, } \\
\text { 1/2 CTC }\end{array}$} & $\begin{array}{l}(1: 3: 3) \\
\end{array}$ & 4,223 & 2,111 & 0 & 0 \\
\hline & & $(1: 3: 1)$ & 12,670 & 6,335 & 0 & 0 \\
\hline & \multirow{2}{*}{$\begin{array}{l}\text { 16 QAM, } \\
\text { 3/4 CTC }\end{array}$} & $(1: 3: 3)$ & 6,336 & 3,168 & 0 & 0 \\
\hline & & $(1: 3: 1)$ & 19,01 & 9,505 & 0 & 0 \\
\hline & \multirow{2}{*}{$\begin{array}{l}\text { 64 QAM, } \\
\text { 5/6 CTC }\end{array}$} & $(1: 3: 3)$ & 10,56 & 5,280 & 0 & 0 \\
\hline & & $(1: 3: 1)$ & 31,680 & 15,840 & 0 & 0 \\
\hline \multirow[t]{8}{*}{$35: 12$} & \multirow{2}{*}{$\begin{array}{l}\text { QPSK, } 1 / 2 \\
\text { CTC, } 6 \mathrm{x}\end{array}$} & $(1: 3: 3)$ & 0,266 & 0,133 & 0,057 & 0,029 \\
\hline & & $(1: 3: 1)$ & 0,798 & 0,399 & 0,170 & 0,085 \\
\hline & \multirow{2}{*}{$\begin{array}{l}\text { 16 QAM, } \\
\text { 1/2 CTC }\end{array}$} & $(1: 3: 3)$ & 3,383 & 1,692 & 0,680 & 0,340 \\
\hline & & $(1: 3: 1)$ & 10,151 & 5,076 & 2,040 & 1,020 \\
\hline & \multirow{2}{*}{$\begin{array}{l}\text { 16 QAM, } \\
\text { 3/4 CTC }\end{array}$} & $(1: 3: 3)$ & 5,084 & 2,542 & 1,020 & 0,510 \\
\hline & & $(1: 3: 1)$ & 15,254 & 7,626 & 3,061 & 1,531 \\
\hline & \multirow{2}{*}{$\begin{array}{l}\text { 64 QAM, } \\
\text { 5/6 CTC }\end{array}$} & $(1: 3: 3)$ & 8,486 & 4,243 & 1,700 & 0,850 \\
\hline & & $(1: 3: 1)$ & 25,458 & 12,729 & 5,102 & 2,551 \\
\hline \multirow[t]{8}{*}{$26: 21$} & \multirow{2}{*}{$\begin{array}{c}\text { QPSK, 1/2 } \\
\text { CTC, 6x }\end{array}$} & $(1: 3: 3)$ & 0,193 & 0,096 & 0,113 & 0,057 \\
\hline & & $(1: 3: 1)$ & 0,597 & 0,299 & 0,340 & 0,170 \\
\hline & \multirow{2}{*}{$\begin{array}{l}\text { 16 QAM, } \\
\text { 1/2 CTC }\end{array}$} & $(1: 3: 3)$ & 2,509 & 1,256 & 1,360 & 0,680 \\
\hline & & $(1: 3: 1)$ & 7,528 & 3,764 & 4,081 & 2,041 \\
\hline & \multirow{2}{*}{$\begin{array}{l}\text { 16 QAM, } \\
\text { 3/4 CTC }\end{array}$} & $(1: 3: 3)$ & 3,772 & 1,886 & 2,040 & 1,020 \\
\hline & & $(1: 3: 1)$ & 11,318 & 5,659 & 6,122 & 3,061 \\
\hline & \multirow{2}{*}{$\begin{array}{l}\text { 64 QAM, } \\
\text { 5/6 CTC }\end{array}$} & $(1: 3: 3)$ & 6,299 & 3,150 & 3,401 & 1,701 \\
\hline & & $(1: 3: 1)$ & 18,898 & 9,449 & 10,204 & 5,102 \\
\hline
\end{tabular}

\section{III.2. CAPACIDADE DE CHAMADAS VOIP}

A capacidade de chamadas VoIP depende do codec de voz utilizado. Na tabela VI tem-se a quantidade de bytes no “paylod” do pacote VoIP para os codecs G.711, G.729 e G.726 para 20ms de voz e a taxa de bits considerando-se um “overhead” de 40 bytes (cabeçalho RTP/UDP/IP no IPv4).

TABELA VI

TAXA DE BITS POR CODEC

\begin{tabular}{|c|c|c|}
\hline Codecs & Payload (bytes) & Taxa de bits (kbps) \\
\hline G.711 & 160 & 80 \\
\hline G.726 & 40 & 32 \\
\hline G.729 & 20 & 24 \\
\hline
\end{tabular}

Nesta seção, é calculada a quantidade máxima de chamadas VoIP utilizando-se ErtPS para os codecs mostrados na tabela VI. Adicionalmente, são feitas as seguintes suposições:

- $\quad$ Largura de banda: $10 \mathrm{MHz}$

- $\quad$ Reuso de freqüência: (1:3:3)

- Modulação: QPSK, 1/2 CTC, 6x, 16 QAM, 1/2 CTC, 16 QAM 3/4 CTC e 64 QAM, 5/6 CTC

- $\quad$ DL:UL 26:21
Das equações (1) e (4), obtém-se que a quantidade de chamadas VoIP no enlace direto é dado por:

$$
N_{D L}=\frac{s p_{-} d l}{d \times s} \times n b\left(t s_{-} d l-1,497\right) /\left(t b_{-} d l u+0,13 \times \frac{s p \_d l}{s \times d}\right)
$$

onde $t b \_d l u$ é a taxa de bits do codec no enlace direto conforme mostrado na tabela VI.

Analogamente, das equações (2) e (5), obtém-se que a quantidade de chamadas VoIP no enlace reverso é dado por:

$$
N_{U L}=\frac{s p_{-} u l}{d} \times n b \times \frac{t s_{-} d l-3}{s \times t b_{-} u l u}
$$

onde $t b \_u l u$ é a taxa de bits do codec no enlace reverso conforme mostrado na tabela VI.

Na tabela VII são apresentados o número máximo de chamadas VoIP simultâneas por tipo de codec e esquema de codificação e modulação. Devido ao “overhead” do pacote VoIP, o aumento no número de chamadas VoIP em função do tipo de codec é significativamente menor do que o fator de redução da taxa de bits. Por exemplo, utilizando-se o codec G.729, embora a taxa de bits seja 8 vezes menor do que a taxa de bits do codec G.711, o aumento no número de chamadas VoIP simultâneas é de 2,5 a 3,3 vezes. Para minimizar este efeito do "overhead" do pacote VoIP na capacidade do IPOFDMA, podem ser utilizados esquemas de compressão do “overhead” [10, 11]. Por ouro lado, o número de chamadas VoIP é também significativamente afetado pelo esquema de codificação e modulação utilizado.

\section{TABELA VII}

NÚMERO DE CHAMADAS VOIP SIMULTÂNEAS

\begin{tabular}{|c|c|c|c|c|c|c|}
\hline \multirow{2}{*}{ Codecs/Modulações } & \multicolumn{2}{|c|}{$\mathrm{G} .711$} & \multicolumn{2}{c|}{ G.726 } & \multicolumn{2}{c|}{ G.729 } \\
\cline { 2 - 7 } & $N_{\mathrm{DL}}$ & $N_{\mathrm{UL}}$ & $N_{\mathrm{DL}}$ & $N_{\mathrm{UL}}$ & $N_{\mathrm{DL}}$ & $N_{\mathrm{UL}}$ \\
\hline QPSK, 1/2 CTC, 6x & 2 & 2 & 4 & 5 & 5 & 6 \\
\hline 16 QAM, 1/2 CTC & 24 & 22 & 47 & 55 & 55 & 73 \\
\hline 16 QAM, 3/4 CTC & 36 & 33 & 70 & 82 & 83 & 109 \\
\hline 64 QAM, 5/6 CTC & 60 & 55 & 117 & 137 & 139 & 182 \\
\hline
\end{tabular}

\section{ANÁLISE DE COBERTURA}

Tendo em vista a forte influência do esquema de codificação e modulação na capacidade do IP-OFDMA, nesta seção é feita uma análise de cobertura do sistema utilizando-se o modelo "shadowing” dado por [6]:

$$
\left[\frac{P_{r}(d)}{P_{r}\left(d_{0}\right)}\right]_{d B}=-10 \beta \log \left(\frac{d}{d_{0}}\right)+X_{b}
$$

onde, $P_{r}(\mathrm{~d})$ é a potência medida, $P_{r}\left(\mathrm{~d}_{0}\right)$ é uma potência de referência e $\beta$ é um fator de perda de propagação. O parâmetro $d$ é a distância da medida em relação à $\mathrm{BS}, d_{0}$ é uma distância de referência e $X_{\mathrm{b}}$ é uma distribuição log-normal. 
Na fugura 4 é mostrada a característica de propagação do IPOFDMA em $2,5 \mathrm{GHz}$ para um valor de $\beta=5,2$ que caracteriza um ambiente bastante obstruído e $d_{0}=1 \mathrm{~m}$. Observa-se que a área de cobertura de altas taxas de bits ou alta capacidade concentra-se em torno da BS até uma distância de $120 \mathrm{~m}$. A área de cobertura de baixa capacidade concentra-se na periferia da célula, mas representam uma porcentagem significativa da área de cobertura. Assim, de acordo com os resultados mostrados na tabela VII, um único usuário VoIP ativo com codec G.711 na região periférica usará toda uma banda de 3,3 $\mathrm{MHz}$.
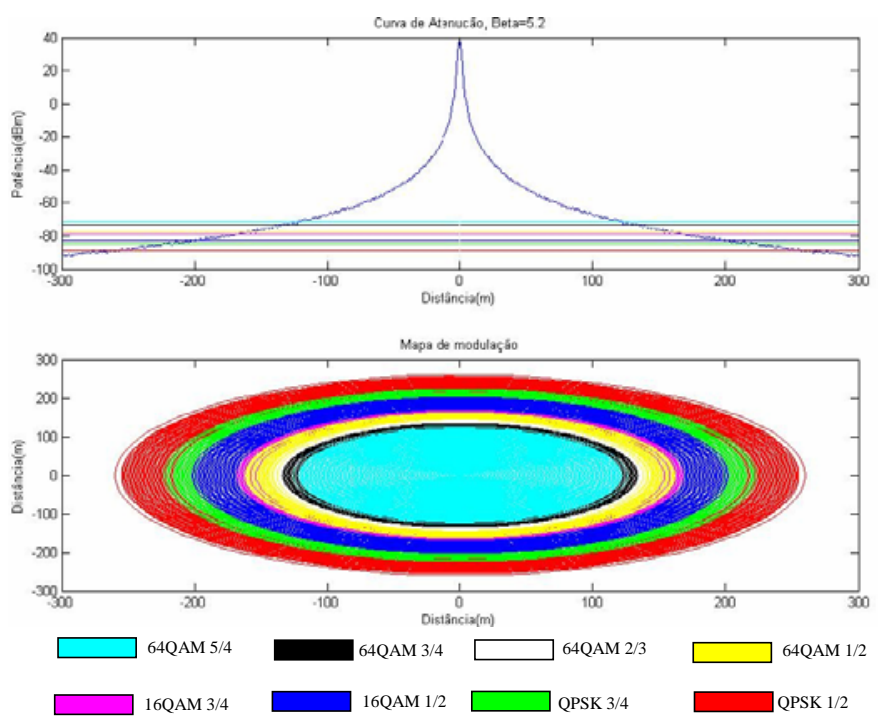

Fig.4. Característica de cobertura do IP-OFDMA

\section{CONCLUSÕES}

Neste trabalho foi feita uma análise de capacidade e desempenho do IP-OFDMA em 2,5 GHz, proposto pelo IEEE e WiMAX Fórum como uma nova tecnologia de transmissão rádio IMT-2000. Foram analisados as taxas de bits por canal de RF e capacidade de chamadas VoIP. Os resultados de capacidade mostram que o IP-OFDMA atende os requisitos do IMT-2000. Entretanto, uma análise de desempenho em termos de cobertura, utilizando-se o modelo "shadowing", mostra que a capacidade da célula é comprometida pelos usuários situados na periferia da célula da mesma. De acordo com a simulação realizada, esta região de baixa capacidade corresponde à uma porcentagem significativa da cobertura da célula. Adicionalmente, devido ao "overhead" do pacote VoIP, para obter um aumento significativo na capacidade do IP-OFDMA com o uso de codecs de voz a baixas taxas, deve-se utilizar técnicas de compressão de "overhead” do pacote VoIP.

\section{Agradecimentos}

Os autores agradecem o suporte do laboratório WCN ("Wireless Competence Network") da INTEL do Instituto de Computação da UNICAMP. Agradecem também à ANATEL pela oportunidade dada em estudar os regulamentos da tecnologia IP-OFDMA como interface radio.

\section{REFERÊNCIAS}

[1] "Air Interface for Fixed Broadband Wireless Access Systems,” IEEE STD 802.16 - 2004, October, 2004.

[2] "Air Interface for Fixed and Mobile Broadband Wireless Access Systems,” IEEE Std 802.16e/D12, February, 2005.

[3] Hassan Yagoobi, "Scalable OFDMA Physical Layer in IEEE 802.16 WirelessMAN”, Intel Technology Journal, Vol 08, August 2004.

[4] Circular Letter 8/LCCE/153: "Announcement of the submission of a candidate radio interface technology for consideration for inclusion in Rec. ITU-R M.1457 as the sixth radio interface in the IMT-2000 terrestrial radio interfaces under the 8/LCCE/95 process".

[5] Document 8F/1079-E: "Additional Technical Details Supporting IP-OFDMA as an IMT-2000 Terrestrial Radio Interface”, 10 de Janeiro de 2007.

[6] Goes, A.; Branquinho, O. C; Reggiani, N. "Effect of Flat Fading in 802.11 MAC for Cross Layer Evaluation Using Channel Emulator”. In: WOWMOM, 2007- Helsinki Finland.

[7] WiMAX Forum, "Mobile WiMAX - Part I: A Technical Overview and Performance Evaluation", 2006.

[8] T. Kwon et al., "Design and implementation of a simulator based on a cross-layer protocol between MAC and PHY layers in a WiBro compatible IEEE 802.16e OFDMA system,” IEEE Communications Magazine, Vol. 43(2), pp. 136-146, Dec. 2005.

[9] WiMAX Forum, "Evaluation of Maximum Number of VoIP Calls over WiMAX Networks with WiBro Profile Version 2.0", March, 2007.

[10] D. Taylor et al. "Robust Header Compression (ROHC) in Next-Generation Network Processors", IEEE/ACM Transactions on Networking, Vol. 13 no. 4, pp 755-768, August, 2005.

[11] W. Chen et al. "Enhancing CRTP by retransmission for wireless networks", In. Proc. of International Conference on Computer Communications and Networks, pp. 426431, Oct. 2001. 